\title{
The variable functional effects of the pacing site in normal and scarred ventricles
}

\author{
Luisa Muñoz del Romeral, MD, PhD, a Carol Stillson, BA, RVT, ${ }^{\mathrm{b}}$ Michael Lesh, \\ $M D,{ }^{c}$ and Elias Botvinick, $M D^{b}$
}

Background. The pacing site has been shown to influence functional improvement with cardiac resynchronization therapy. We evaluated the effects of the pacing site on left ventricular (LV) function in an animal model.

Methods and Results. Equilibrium radionuclide angiography was acquired in sinus rhythm (NSR) and with ventricular pacing, from three pacing sites in seven normal and eight infarcted dogs. QRS duration, electrical activation pattern, wall motion, LV ejection fraction (EF), synchrony of ventricular contraction, and mean arterial pressure (MAP), were related to the pacing site and infarct size, during each of 120 episodes. Little changed during pacing in normals. In infarcted dogs, LV wall motion, and synchrony worsened, LVEF and MAP often fell. These changes related to altered activation patterns which were influenced by the pacing site but were not related to infarct size.

Conclusions. Hemodynamic and functional $\mathrm{LV}$ changes after infarction were found to vary with the pacing site and associated conduction and synchrony. (J Nucl Cardiol 2009;16:904-13.)

Key Words: Ventricular function • pacing • electrophysiology $\bullet$ synchrony

\section{INTRODUCTION}

The placement of biventricular pacemakers, cardiac resynchronization therapy (CRT) is widely applied with benefit to patients with severe left ventricular (LV) systolic dysfunction, LBBB, and refractory heart failure (HF) ${ }^{1-4}$ Yet, there is no clinical benefit in as many as $40 \%$ of CRT patients. ${ }^{3}$ In this setting, different pacing sites, present varied conduction and contraction sequences, related to varying functional responses ${ }^{5}$ and clinical benefit. The outcome is likely to be affected by

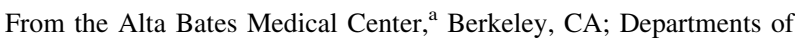
Medicine and Radiology, Cardiovascular Division, Cardiovascular Research Institute, ${ }^{\mathrm{b}}$ University of California San Francisco, San Francisco, CA; Evera Medical, Inc, ${ }^{\mathrm{c}}$ Foster City, CA.

Supported by a Research Fellowship from the American Heart Association, California Affiliate.

Received for publication Mar 16, 2009; final revision accepted Jul 25, 2009.

Reprint requests: Elias Botvinick, MD, Departments of Medicine and Radiology, Cardiovascular Division, Cardiovascular Research Institute, University of California San Francisco, 505 Parnassus Avenue, Box 0214, San Francisco, CA 94143; botvinicke@medicine. ucsf.edu.

1071-3581/\$34.00

Copyright (C) 2009 The Author(s). This article is published with open access at Springerlink.com.

doi:10.1007/s12350-009-9135-4 the presence and location of scar. Optimal outcome is thought to relate to pacemaker placement in the latest contracting LV segment, generally thought to be the lateral $\mathrm{LV}$ wall in LBBB. ${ }^{5,6}$ However, the functional effects of varying pacemaker location have not been well documented. We evaluated the effects of pacing site on conduction, systolic function, synchrony of ventricular contraction, and mean arterial pressure (MAP), in the absence and presence of scar.

Equilibrium radionuclide angiography (ERNA) was applied for its objectivity, reproducibility, quantitative nature, portability, its ability to display the contraction sequence, and assess synchrony. ${ }^{7-11}$

\section{METHODS}

\section{Experimental Design}

We studied 16 healthy, 24-35 kg mongrel dogs under institutional and NIH guidelines. Seven were studied at baseline and nine were studied 1 month after production of a myocardial infarction. One infarcted animal was excluded when it became unstable during study.

Post-absorptive dogs were pre-medicated with morphine sulfate and anesthetized with alpha chloralose. Animals were intubated, ventilated, hydrated, thermoregulated, and continuously monitored with a surface electrocardiogram. An abdominal aorta catheter continuously monitored blood pressure. 


\section{Surgical Procedure}

The heart was exposed through a median thoracotomy and placed in a pericardial cradle. Pacing electrodes and a monitoring sock array were positioned on the myocardium. The proximal terminals of the electrodes were exteriorized and the thoracotomy closed. The animals were later paced in multiple sessions, from three positions: high septum (HS), right ventricular free wall (RVW), and LV apex (LVa).

A ligation of the mid-left anterior descending coronary artery (LAD) beyond the first diagonal vessel by the Harris procedure $^{12}$ produced a small, distal anteroseptal infarction. All visible collaterals were ligated.

ERNA was acquired in the anterior and LAO views during sinus rhythm (NSR) and with each pacing episode, to determine LV wall motion, calculate LV ejection fraction $(\mathrm{EF})$, mean LV and right ventricular (RV) phase angles $(\varnothing)$, and the standard deviations (SD) of LVØ and RVØ. Blood pressures were averaged and expressed as MAP: systolic blood pressure-2(diastolic blood pressure)/3. After the protocol, infarcted hearts were infused with saturated triphenyltetrazolium chloride (TTC) solution immediately before killing. After barbiturate euthanasia, excised hearts were preserved in formalin.

\section{Pacing Protocol}

Bipolar epicardial pacing was performed in random order through three silver wire electrodes sutured to the HS, RVW, or LVa, at a $300 \mathrm{~ms}$ cycle length at twice the stimulation threshold to capture the ventricles. Simultaneous right atrial pacing abolished the atrial contribution to LV filling. Pacing for 10 minutes permitted complete and stable data acquisition. Ten minutes between pacing episodes achieved full recovery. A 56 bipolar electrode band array or "sock" (C.R. Bard, Inc., Tewksbury, MA), with 14 columns of 4 bipoles, each with 2 electrode contacts $2 \mathrm{~mm}$ apart evenly distributed around the ventricles, recorded epicardial activation electrograms. The LAD position was the $0^{\circ}$ polar marker applied to orient the "sock'. Pacing wire position was related to the closest band array bipolar electrode in a diagram of the sock. During pacing, bipolar electrograms and eight surface electrocardiograms were simultaneously recorded and digitized (BARD 64 Cardiac Mapping System, v.B.05), with generation of epicardial isochronic maps and measurement of QRS duration for each pacing episode. The earliest ventricular depolarization site was compared to the anatomic pacing site and the site of earliest phase angle, and was related to the associated epicardial electrical activation sequence and the pattern of phase angle progression.

\section{ERNA}

Acquisition and processing. ERNAs were acquired in 16 frames in the standard manner, on a Siemens portable scintillation camera (Siemens USA, Des Plaines, IL) using a low-energy all purpose collimator and processed on a PDP 11/40 minicomputer (Digital Equipment Corp., Maynard,
MA). Subject red cells were labeled in vitro with approximately $30 \mathrm{mCi}$ of technetium-99m. ${ }^{13}$ ECG gated images were acquired and digitized in NSR, and with all pacing episodes, in the anterior and "best septal" LAO views, acquired with $15^{\circ}$ caudal angulation, to separate LV from left atrium.

Phase analysis. Phase analysis, generated from the first harmonic fit of the LAO ERNA time versus radioactivity curve, marked the timing of regional ventricular contraction. ${ }^{8}$ It permitted visualization of the sequence and estimation of the duration of ventricular contraction, and by association, activation and excitation. ${ }^{8-10}$ Phase images were gray scale coded for determination of serial phase angle progression, ${ }^{8}$ in increments of $2.8^{\circ}$. The difference, mean LV $\varnothing-$ mean RV $\varnothing$, served as a surrogate for interventricular synchrony, and the SD LVØ and SD RVØ, were respectively, surrogates for LV and RV intraventricular synchrony, each measured blindly.

\section{Wall Motion}

Analysis. Segmental wall motion was blindly assessed in six regions: apex; septum; anterior, lateral, and inferior LV; and RV free wall. ${ }^{7,8}$ Two experienced observers agreed upon wall motion graded visually as: normal; hypokinetic, with reduced systolic motion; akinetic, without motion; and dyskinetic, with paradoxical systolic motion. Isolated dyskinesis was consistent with aneurysm. Ventricles were categorized based on the most abnormal wall motion grade identified by consensus of the two readers.

Reproducibility. In order to demonstrate the extent and limits of analytic reproducibility, two readers blindly read wall motion in six segments in each of 16 randomly selected ERNA images performed in 4 random post-infarction dogs. Wall motion analysis was in complete agreement in 92 of 96 segments with disagreement of one grade in only four abnormal segments.

LVEF was calculated blindly by the standard method from manually drawn LV regions of interest, from the LAO ERNA images with each pacing episode. When multiple repeat measurements of LVEF were performed by the same observer, LVEF varied $\pm 5 \%$, which represented two standard deviations from the mean, slightly greater than for the same calculations in patient studies. ${ }^{7}$

\section{Infarct Size}

After fixation, transverse LV sections from base to apex were photographed on both apical and basal surfaces and slice thickness, expressed as the fraction of the ventricle, was measured. The areas of non-viable regions demarcated by TTC staining, as well as the entire LV area, were manually planimetered (Planix 7, Tamaya, Japan) by two independent, blinded operators using enlarged photographs of each section. The average infarct area on basal and proximal surfaces of each slice was divided by the average of total basal and proximal slice surface to yield a percentage infarct for each slice. These per slice averages were weighted for slice thickness and summed for all slices. The percentage LV infarcted (\% infarct) was calculated as: 


$$
\% \text { Infarct }=\sum_{1}^{k} \frac{(\text { apical scar area }+ \text { basal scar area }) / 2_{n} \times(\text { section thickness })_{n}}{(\text { apical section area }+ \text { basal section area }) / 2_{n}} \times 100
$$

where $k=$ number of ventricular sections, section thickness is expressed as the percentage of the LV length and $n$ is the number of the LV section. Small infarcts were $\leq 5 \%$, medium if $>5 \%$ but $<10 \%$, and large if $\geq 10 \%$ of total LV mass. All image, hemodynamic, electrophysiologic, and pathologic analysis was blinded to other findings and the results of serial studies.

The authors had full access to the data and take responsibility for its integrity. All authors have read and agree to the manuscript as written.

\section{STATISTICAL ANALYSIS}

All results are expressed as mean $\pm \mathrm{SD}$. Two-way analysis of variance analyzed changes in MAP related to pacing site, LV wall motion and LVEF, and infarct size, and compared differences between normal and infarcted dogs. Bonferroni and Dunnet's tests isolated between groups differences and permitted multiple comparisons. The Chi square test related individual pacing sites to the functional response, LV wall motion abnormalities, LVEF, and phase parameters. The sample size for all calculations was the first of two mapping studies for each of seven normal dogs and eight with infarction as noted in "Results" below.

\section{RESULTS}

\section{Electrophysiologic and Phase Data}

Reproducibility. Data sets generated during multiple pacing episodes from the same site brought little change, no greater than the intraobserver variability. The initial episode was used in all calculations.

The region of earliest ventricular depolarization and earliest phase angle, corresponded to the anatomical pacing site in all experiments.

Normal dogs. In 7 normal dogs, 56 episodes were studied, 8 episodes per dog, 2 in NSR and at each pacing site with 14 episodes in NSR and 42 pacing episodes.

During NSR, the electrical impulse spread rapidly and simultaneously through both ventricles from base to apex, with the LV first to depolarize. The QRS complex was 60 milliseconds with SD LVØ $\left(10.9^{\circ} \pm 6.2^{\circ}\right)$, SD $\mathrm{RV} \varnothing\left(11.2^{\circ} \pm 5.8^{\circ}\right)$, and mean $\mathrm{LV} \varnothing-$ mean $\mathrm{RV} \varnothing$ $\left(6.5^{\circ} \pm 3.6^{\circ}\right)$.

During HS pacing, electrical activation spread rapidly to both ventricles, RV sometimes earlier than LV, with an insignificant conduction delay and QRS lengthening (Figures 1A and 1B).

During RVW pacing, electrical activation spreads from RV-free wall to RV body, from base to septum and then to the entire LV in concentric waves. During LVa pacing, activation spread from LV apex to base in concentric waves encompassing both LV and RV.

Compared to NSR, both RVW and LVa pacing brought a prolongation of ventricular activation, with lengthening of the QRS duration to 120 millisecond, $P<.001$. RVW and LVa pacing brought non-significant delays in the earliest and mean LVØ and RVØ.

Infarcted dogs. In 8 infarcted dogs, 64 episodes were studied, 8 episodes per dog, 2 in NSR and 2 at each pacing site, with 16 episodes (Figures 1C and 1D) in NSR and 48 paced episodes.

During NSR, the activation again spread rapidly through the ventricles in an organized manner from base to apex, with the LV first to depolarize. The QRS complex was not lengthened compared to normals and $\operatorname{SD~LV} \varnothing\left(11.9^{\circ} \pm 9.1^{\circ}\right)$, SD RVØ $\left(12.0^{\circ} \pm 6.9^{\circ}\right)$, and mean $\operatorname{LV} \varnothing-$ mean $\operatorname{RV} \varnothing\left(6.1^{\circ} \pm 4.2^{\circ}\right)$ were not significantly different from those values in normal dogs.

All phase parameters increased significantly with each pacing site compared to those measured in association with the same pacing site in normal dogs, $P<.05$. During HS pacing, electrical activation progressed as in normals but with a terminal delay in the peri-infarct area. HS pacing brought lengthening of neither ventricular activation nor of the QRS. Compared with NSR, SD $\operatorname{LV} \varnothing\left(18.5^{\circ} \pm 5.2^{\circ}\right), \operatorname{SDRV} \varnothing\left(17.5^{\circ} \pm 7.0^{\circ}\right)$, and mean LV $\varnothing-$ mean RV $\varnothing\left(8.9^{\circ} \pm 6.2^{\circ}\right)$ all increased significantly, $P<.05$ versus NSR.

During RVW pacing activation spread from the distal RV-free wall to the RV body and then to the LV in concentric waves with varying delay in the peri-infarction region (Figures $1 \mathrm{C}$ and $1 \mathrm{D}$ ). $\mathrm{RV}$ pacing lengthened ventricular activation and QRS duration (120 millisecond), $P<.05$ versus both NSR and HS pacing, and increased SD LVØ $\left(25.8^{\circ} \pm 16.4^{\circ}\right)$, SD RVØ $\left(21.5^{\circ} \pm\right.$ $\left.5.7^{\circ}\right)$, and mean $\mathrm{LV} \varnothing-$ mean $\mathrm{RV} \varnothing\left(11.4^{\circ} \pm 12.2^{\circ}\right)$, all $P<.05$ versus NSR.

During LVa pacing, activation was most aberrant, spreading from the LV apex proximally to both ventricles, bypassing the usually anterior-apical peri-infarct area, which was often activated late. LVa pacing lengthened ventricular activation and QRS duration 

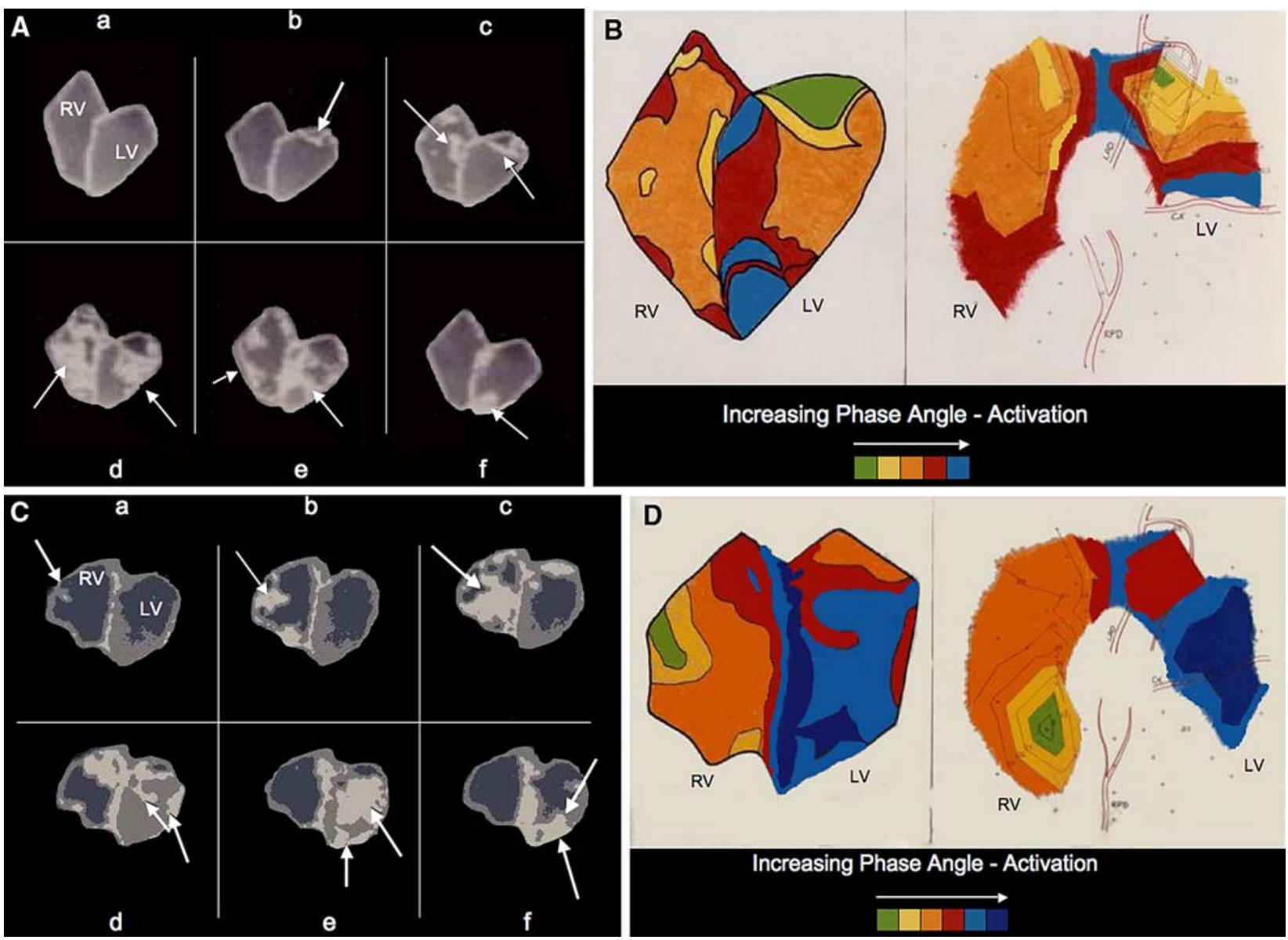

Figure 1. Phase and isochronous epicardial maps. Shown are serial phase images with masked background, in a normal animal studied with HS pacing (A) and in an infarcted animal with RVW pacing $(\mathbf{C})$. Progressive whitening of the phase images in panels (a-f) of these figures delineates the course of progression of serial phase angle and related contraction through right, RV, and left, LV, ventricles. Arrows in panels ( $\mathrm{a}$ and $\mathrm{b}$ ) of each figure localize the site of earliest phase angle. Subsequent arrows point to areas of sequential phase angle progression. The left panels of $\mathbf{B}$ and $\mathbf{D}$ are diagrammatic color coded phase maps which summarize the serial phase progression of the phase image in the figures they follow, 1B with HS pacing and 1D with RVW pacing of an infarcted ventricle. Here the color progression, shown in the color scale at the bottom of each figure, parallels the phase progression highlighted in white on the preceding figures. The right panels of $\mathbf{B}$ and $\mathbf{D}$ present the related color coded isochronous epicardial maps, which summarize the serial activation pattern, here related to their associated phase maps, at left, drawn in the anatomically opened RV and LV, with walls exposed. The coronary distribution is roughly illustrated by the $L A D$ left anterior descending coronary artery, $C X$ left circumflex coronary artery, and $R P D$ right posterior descending coronary artery. In each case, serial phase progression related well to the epicardial activation sequence. In the normal ventricle, $\mathbf{A}$ and $\mathbf{B}$, phase angle progressed through contiguous $\mathrm{LV}$ regions. In contrast phase images $(\mathbf{C})$, summarized in the left panel of $(\mathbf{D})$, in the animal with a distal LAD infarction, reveal initially contiguous phase progression, arrows, panels c and d, until near the infarct area. Here myocardial contraction "skips", a distal myocardial region between the arrows in panel (e). This region demonstrates delayed phase progression, arrow, panel (f). A similar pattern was well seen on the activation map, $\mathbf{D}$, right panel, with some variation.

(120 millisecond), $P<.05$ versus NSR and HS, and increased the $\operatorname{SD~} \operatorname{LV} \emptyset\left(40.5^{\circ} \pm 21.3^{\circ}\right), P<0.01$ versus NSR, HS, and RVW, SD RVØ $\left(18.6^{\circ} \pm 6.1^{\circ}\right), P<.05$ versus NSR, and mean LVØ - mean RVØ $\left(22.1^{\circ} \pm 16.5^{\circ}\right), P<.05$ versus NSR, HS, and RVW.

\section{MAP}

Normal dogs. MAP decreased moderately at all pacing sites (Figure 2). Compared to NSR, RVW pacing had the largest fall in MAP, $19.1 \%(P<.02)$, with 


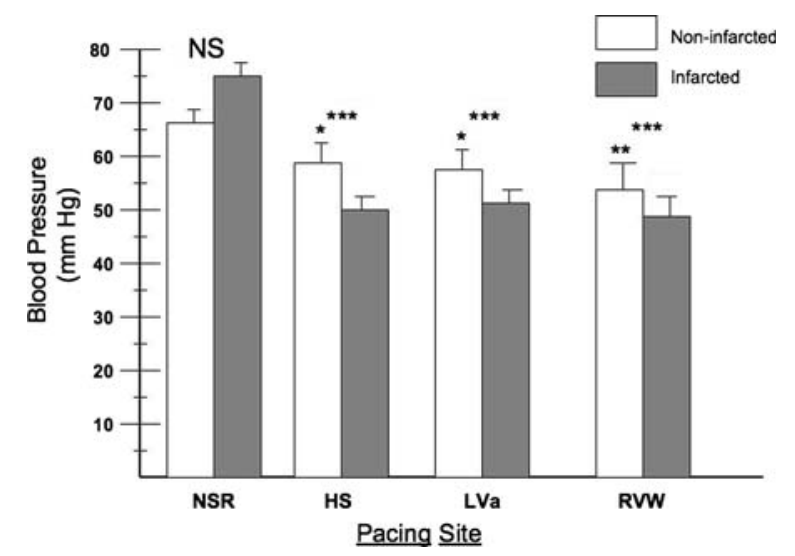

Figure 2. MAP and pacing site. Shown is the comparison of MAP, blood pressure, between normal and infarcted dogs in NSR and at each pacing site. During NSR MAP in infarcted and normal dogs was not significantly different (NS). MAP was not significantly different between pacing sites in either normal or infarcted dogs. However, MAP decreased at all pacing sites compared to NSR in the same normal dogs, $* P<.05$, ** $P<.02$, and was lower at all pacing sites in infarcted compared to normal dogs, $* * * P<.01$.

$12.4 \%$ and $11.7 \%$ reductions in MAP on LVa and HS pacing, respectively (both $P<.05$ ).

Infarcted dogs. During NSR MAP was not significantly different from normal dogs. Although MAP in infarcted dogs was not significantly different between pacing sites (Figure 2), MAP decreased at all sites compared to NSR in the same animal $(P<.0001)$, and was lower at all pacing sites compared to normal dogs $(P<.01)$.

\section{Wall Motion and LVEF}

Normal dogs. Wall motion and LVEF remained essentially normal during pacing from all sites with the exception of reduced septal motion in three dogs with RVW pacing.
Infarcted dogs. In each animal, the type and severity of LV wall motion abnormalities varied with the pacing site. Wall motion in the infarcted group was normal in 20 episodes of which $13(65 \%)$ were in NSR, $6(30 \%)$ during HS pacing, and $1(5 \%)$ during RVW pacing. Mild wall motion abnormalities with hypokinesis were seen in 25 episodes, of which 3 (12\%) were in NSR, 9 (36\%) during HS pacing, 9 (36\%) during RVW pacing, and 4 (16\%) during LVa pacing. Akinetic segments were seen in two episodes, one during HS pacing and the other during RVW pacing. Dyskinetic, aneurysmal segments were seen in 17 episodes, 5 (29\%) during RVW pacing and $12(71 \%)$ related to LVa pacing $(P<.0001)$. The LVa pacing site produced the most severe wall motion abnormalities with the greatest increase of mean $\varnothing$ and SDØ, with the characteristic phase delay seen in aneurysms ${ }^{11}$ (Table 1). Yet, the same animals which demonstrated dyskinetic, dyssynchronous aneurysms during LVa pacing, often had normal or only hypokinetic LVWMA when paced from the HS or RVW (Figure 3, Table 2).

LVEF varied significantly with pacing site, roughly in parallel with generated wall motion abnormalities (Table 3). LVEF fell significantly at all pacing sites when compared with NSR, and was mildly reduced in association with HS pacing, $51.6 \pm 18.0$, moderately reduced with RVW pacing, $43.0 \pm 17.6$, and severely reduced with LVa pacing, $35.8 \pm 17.5$ (LVa vs HS, $P<.01$, LVa vs RVW, $P<.05$, all $P<.01$ vs NSR).

\section{MAP Relationship to Wall Motion}

MAP was lower among animals with LV segmental wall motion abnormalities than among those with normal wall motion, $P<.0001$ (Figure 4). MAP was lowest, but not significantly so, in the small number with akinetic segments. MAP was significantly lower in animals with dyskinetic, dyssynchronous segments, primarily those paced from an LVa site, when compared

Table 1. Wall motion and pacing site-overview

\begin{tabular}{lccccc}
\hline \multicolumn{5}{c}{ Wall motion } \\
\hline Rhythm/pacing site & Normal & Hypokinesis & Akinesis & Dyskinesis/aneurysm & Total \\
\hline NSR & $13^{*}$ & 3 & 0 & 0 & 16 \\
HS & 6 & $9^{*}$ & 1 & 0 & 16 \\
RVW & 1 & $9^{*}$ & 1 & 5 & 16 \\
LVa & 0 & 4 & 0 & $12^{*}$ & 16 \\
Total & 20 & 25 & 2 & 17 & 64 \\
\hline
\end{tabular}

Shown is the relationship between the most severe ERNA LV wall motion abnormality and pacing site among all infarcted dogs. LVa pacing brought the most severe wall motion abnormalities.

${ }^{*} P<.005$ versus other types of wall motion for the same pacing site. 

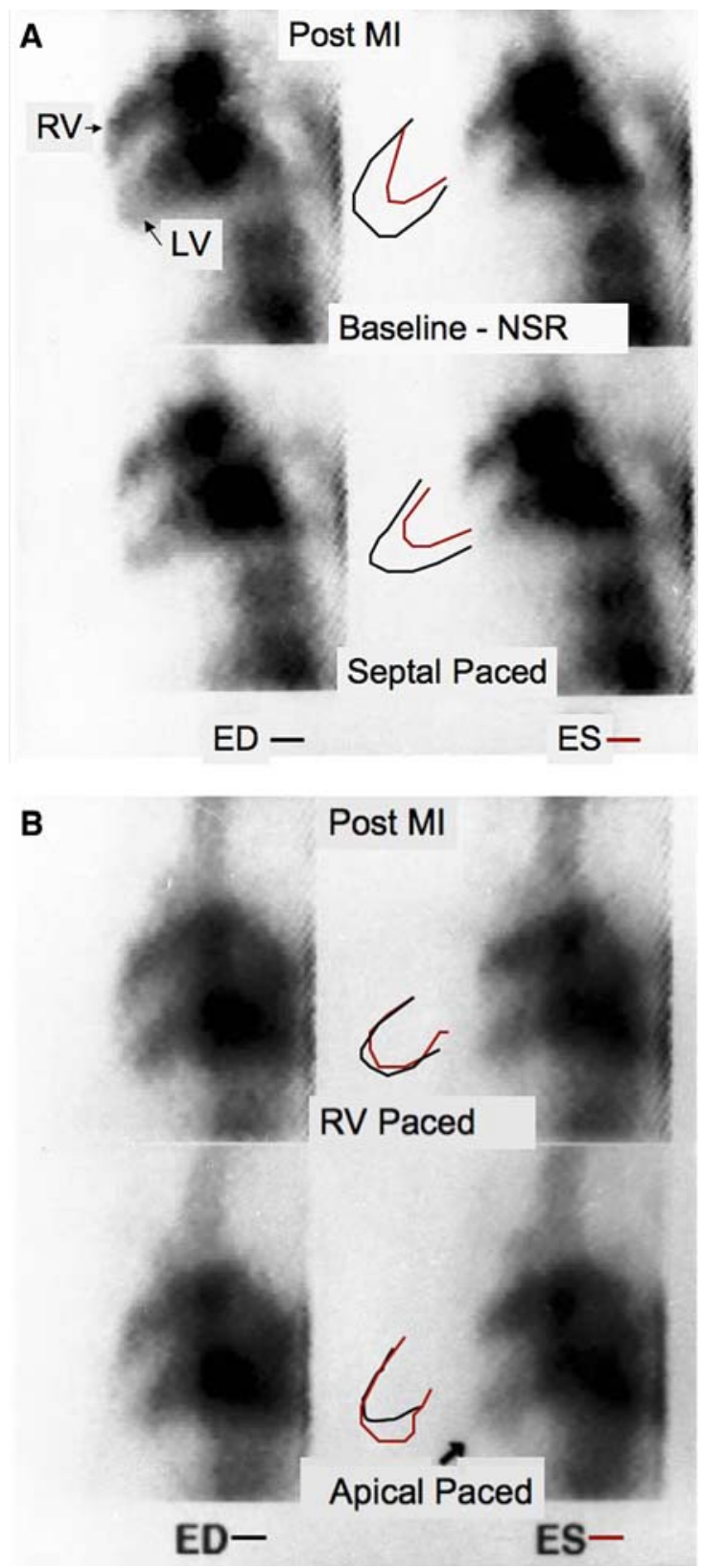

Figure 3. Blood pool images. A Shown are end-diastolic (ED) and end-systolic (ES) frames from the ERNA acquired in an animal with an LAD infarction (MI), during baseline NSR, above, and with septal pacing, below. Normal RV and LV (arrows) wall motion is evident and confirmed by the superimposition of ED (black) and ES (red) contours. B Shown are ED and ES frames from the ERNA acquired in the same animal illustrated in A, now during RV pacing, above, and with LV apical pacing, below. Evident is apical hypokinesis with the RV pacing site, and apical dyskinesis and an apical aneurysm with LVa pacing, arrow. Again, LV wall contours are drawn and superimposed for ED (black) and ES (red), as in $\mathbf{A}$. with animals having normal, $P<.0001$, or hypokinetic wall motion, $P<.02$.

\section{Infarct Size}

All infarcts were in the same distal region including the apex, distal septum, and anterior wall. Infarcts varied in size from approximately $5-15 \%$ of the LV. There were four small, three medium, and a single large infarction. When infarct size was related to the severity of the greatest induced wall motion abnormality in each episode, akinesis or dyskinesis was seen in $50 \%$ of episodes in the dog with a large infarction but was evident too in $28 \%$ of dogs with small infarcts. Given the relatively common occurrence of severe wall motion abnormalities among dogs with small infarcts when paced from specific locations, pathologic infarct size, did not correlate with LV wall motion abnormalities, nor with LVEF or MAP.

\section{DISCUSSION}

\section{Overview}

The pacing site is thought to influence the functional response to CRT. ${ }^{5,14,15}$ However, the functional response to varying pacemaker sites in normal and infarcted ventricles has not been formerly demonstrated. We have applied ERNA to demonstrate the varying functional effects of different pacing modes. ${ }^{9}$ Here, we applied ERNA to determine the functional effects of different pacing sites.

\section{Method}

Under controlled conditions, we evaluated systolic LV function, myocardial synchrony, and MAP, at baseline and with pacing from varying sites in normal ventricles and with a small distal infarction in an animal model. The rate was constant and the influence of varying A-V synchrony, an important influence on paced cardiac output, ${ }^{9}$ was eliminated. Phase imaging objectively displayed the sequence of ventricular contraction, ${ }^{8-11}$ confirmed the conduction sequence and served as an indicator of intra- and interventricular synchrony. ${ }^{9-11}$

\section{Findings}

The study confirms the functional importance of the focus of excitation especially in scarred ventricles. Regional scar has previously been shown to importantly 
Table 2. Wall motion and pacing site in individual infarcted dogs

\begin{tabular}{|c|c|c|c|c|}
\hline \multirow[b]{2}{*}{ Infarcted dog (number) } & \multicolumn{4}{|c|}{ Rhythm/pacing sites } \\
\hline & NSR & HS & RVW & LVa \\
\hline & Wall motion & & & \\
\hline 1 & $\mathrm{NI}$ & Nl/Нypo & Нуро & A/Dys \\
\hline 2 & $\mathrm{NI}$ & Нуро & NI/Hypo & Нуро \\
\hline 3 & Hypo & Нypo/А & A/Dys & A/Dys \\
\hline 4 & NI/Hypo & NI/Hypo & Нyро & A/Dys \\
\hline 5 & $\mathrm{NI}$ & Nl/Hyро & Нуро & A/Dys \\
\hline 6 & $\mathrm{NI}$ & $\mathrm{NI}$ & A/Dys & A/Dys \\
\hline 7 & $\mathrm{NI}$ & Nl/Hypo & A/Dys & A/Dys \\
\hline 8 & $\mathrm{NI}$ & Nl/Hypo & Нуро/А & Нуро \\
\hline
\end{tabular}

Shown are the most severe ERNA LV wall motion abnormalities during the initial episode in NSR and with each pacing site for each infarcted dog. In the same animal wall motion deteriorated frequently and severely with LVa and RVW pacing.

$\mathrm{Nl}$, Normal wall motion; Hypo, hypokinetic wall motion; $A$, akinetic wall motion; Dys, dyskinetic wall motion with aneurysm.

Table 3. Ejection fraction and pacing site in individual infarcted dogs

\begin{tabular}{lllll}
\hline & \multicolumn{4}{c}{ Rhythm/pacing sites } \\
\cline { 2 - 4 } Infarcted dog (number) & NSR & HS & RVW & LVa \\
\hline \multicolumn{4}{c}{ Left ventricular } & ejection fraction (\%) \\
1 & 65 & 58 & 47 & 39 \\
2 & 64 & 55 & 53 & 42 \\
3 & 60 & 42 & 36 & 34 \\
4 & 64 & 52 & 42 & 24 \\
5 & 67 & 48 & 42 & 28 \\
6 & 58 & 48 & 46 & 42 \\
7 & 58 & 52 & 32 & 39 \\
8 & 59 & 58 & 46 & 36 \\
Average & 62 & 52 & 43 & 36 \\
\hline
\end{tabular}

Shown is the LVEF for NSR and at each pacing site in each infarcted dog. LVEF was preserved in NSR but fell with each pacing site, most marked with RVW and LVa pacing.

influence the success of CRT. ${ }^{6,16}$ Not previously demonstrated, is the interaction of scar with different pacing sites, presenting a varying effect on LV function and synchrony. We previously demonstrated the influence of the ventricular tachycardia exit site on the pattern of electrical conduction and resultant systolic ventricular function and synchrony. ${ }^{11}$ We now demonstrate a link between the pacing site with its related electrical conduction pattern, and the variable resultant effect on systolic ventricular function and synchrony, in normal and scarred ventricles.

\section{The Interaction of Activation and Contraction}

How does the pacing site exert its influence on ventricular function? The findings support the influence of a varied activation sequence and conduction pattern derived from each pacing site which result in a specific related myocardial contraction sequence. This sequence relates to a specific contraction pattern, resulting in varying LV systolic function, synchrony, and hemodynamics. The magnitude and direction of the functional effect of pacing relates directly to the induced contraction pattern and related synchrony which is determined, in part, by the pattern of electrical activation. The electrical activation pattern is a product of the pacing site and the state of the intervening myocardium. Owing to the influence of scar on both conduction and contraction, these effects are most prominent in the presence of scar. These findings provide insight into the source of variable outcome in CRT, where scar is generally present and often widespread. Tying activation to contraction in a predictable manner is the fact that the 


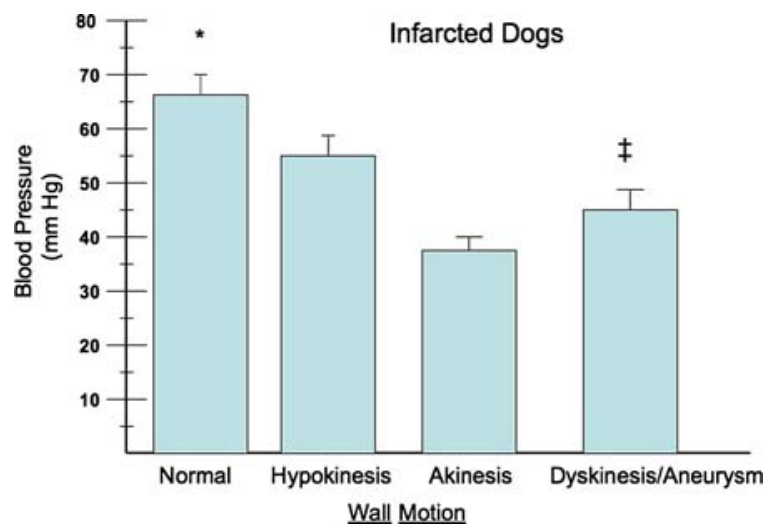

Figure 4. MAP and LVWMA. Shown is the relationship between MAP, blood pressure, and LV wall motion abnormalities so characterized among the eight infarcted dogs. MAP was significantly higher among those with normal wall motion compared to dogs with LV wall motion abnormalities $(* P<.0001)$. MAP was lowest in the group with akinetic segments but their small number prevented significance. MAP was significantly lower in dyskinetic segments $(\$)$, primarily those paced from an LVa site, when compared either with animals having normal $(P<.0001)$ or hypokinetic motion $(P<.02)$.

pattern of electrical activation correlated closely with the mechanical contraction sequence on phase imaging (Figure 1). This correlation is the subject of another manuscript. ${ }^{17}$

\section{Phase Analysis}

Phase analysis previously revealed the prognostic importance of synchrony in congestive cardiomyopathy. ${ }^{10,18}$ The functional benefit of CRT, varies with its ability to improve mechanical synchrony. ${ }^{19-21}$ In this model we demonstrated that the pacemaker site had a widely varying effect on wall motion abnormalities, synchrony, and hemodynamic changes, sometimes bringing improvement, sometimes worsening, to the function of the LV apex, with its limited scar. Decremental MAP during pacing in infarcted dogs, correlated only indirectly with the specific pacing site, but correlated more directly with the presence and severity of LV wall motion abnormalities, and induced dyssynergy, interventricular, and LV intraventricular dyssynchrony. This was most frequent in association with LVa pacing.

The evidence suggests that in this model, LV dysfunction is secondary to delayed conduction through an altered myocardium (Figure 1) producing segmental LV wall motion abnormalities, frequent dyskinesis (Tables 1 and 2) and dyssynchrony. When activation originated distal to the infarction, from the LV apex, LVa pacing, both ventricles demonstrated retrograde depolarization and delayed conduction in the peri-infarct area, producing a dyskinetic, dyssynchronous contraction pattern, decremental function, and reduced MAP. Compared to the more physiologic conduction pattern of HS pacing, the pattern of retrograde ventricular activation related to this LVa pacing site would be expected to encounter the distal infarct area early on, requiring the most circuitous activation sequence to gain access to the bundle branches, with the greatest resultant conduction delay and ventricular dyssynchrony. The delay in activation with LVa compared to HS pacing was clear and the resultant wall motion abnormalities were evident (Figure 3, Tables 1 and 2). Delay in contraction and, presumably, conduction in the peri-infarct region, was also seen in relation to RVW pacing (Figures 1C and 1D). Possibly owing to their temporal resolution, such delay was sometimes seen better on phase than isochronous activation maps.

\section{Relationship to Infarct Size}

The lack of correlation between pathologic MI size and LV wall motion abnormalities or hemodynamics, appears to be due, in part, to the small numbers involved, and the varied distribution of infarct size. It is likely influenced as well, by the variable alteration in conduction through and around scar tissue. This leads to an altered contraction sequence and variable ventricular function which again suggests the importance of the pacemaker site to ventricular function and the relatively small effect of the limited scar present here.

\section{Study Deficiencies}

CRT is delivered with endocardial, A-V synchronous, biventricular pacing. We studied the functional effects of a few representative isolated pacing sites which were chosen with consideration of their likely differences on ventricular function.

Epicardial pacing was applied to gain a selection of stable pacing sites as it is sometimes necessarily applied with $\mathrm{CRT} .^{22}$ Epicardial activation, conduction, and possibly hemodynamic effects may differ from the endocardial location. However, phase image documentation of earliest contraction at the pacing site and the demonstrated relationships of specific contraction patterns and resultant LV function with the designated pacing site, are most important here and not the specific form of pacing. The parallels with and implications for CRT are clear.

Although animal numbers are relatively small, the number of episodes studied is adequate to establish the relationships sought. This intensive protocol terminated with killing of the subject, so we sought to achieve our objective with fewest animals. 
That large infarctions relate to more frequent and more severe wall motion abnormalities is well established. The lack of relationship here between infarct size and LV function is negatively affected by the single large infarction and other factors. Demonstrated here was the frequent occurrence of severe wall motion abnormalities in relation to small infarctions and their variation with the pacing site! These same principles must be demonstrated to apply to humans.

Although overlapping structures could lead to ERNA inaccuracies in both phase and LVEF calculation in dogs, structural separation permitted appropriate regions of interest, adequate reproducibility, and likely accuracy.

Phase angle is generated from a symmetrical cosine curve fit of the asymmetrical regional time versus radioactivity curve for each pixel of the ERNA. A multiharmonic fit of the ERNA time activity curve would increase the accuracy of the curve fit and would be required to translate phase angle into absolute timing of contraction onset. However, a multiharmonic fit would require greater data density than that which is available in a single image pixel and would require analysis of grouped pixels. Yet, multiharmonic fits demonstrated no advantage over the first harmonic method for the evaluation of serial phase or contraction sequence where the sequence of phase angle progression correlated well with electrocardiographic and electrophysiologic methods in this and prior studies. ${ }^{2-10,17}$

Differences in the sampling rate between the phase image and epicardial map presented an intrinsic obstacle to exact agreement of the two. However, the data well supported the comparison of their sequences. In the figures, appropriate intervals were empirically selected to demonstrate the relationship. Illustrations were designed to demonstrate the sequence of phase progression not obscure it.

In this preliminary study, arterial pressure was the only hemodynamic parameter measured. Thus, if hemodynamic changes were induced by reflex mediated mechanisms causing vasodilation or altering venous return, these would not be detected.

\section{CONCLUSIONS}

CRT has been shown to improve LV function, hemodynamics, performance, and quality of life in some, but not all, patients with severe HF. ${ }^{1-4,23-25}$ The mechanism of improvement relates, in part, to less circuitous conduction and improved ventricular synchrony in the presence of conduction abnormalities and scar. ${ }^{6,16,18-21}$ The findings suggest that interventricular and, more so, intraventricular, conduction and related synchrony, influences contraction and related function.
The benefits of pacing are likely largest in those with the greatest baseline intraventricular dyssynchrony. ${ }^{18,19} \mathrm{In}$ addition, the presence of scar, ${ }^{16}$ as well as its location and relationship to the pacing site, also likely influence any improvement in ventricular synchrony and the success of CRT.

\section{Open Access}

This article is distributed under the terms of the Creative Commons Attribution Noncommercial License which permits any noncommercial use, distribution, and reproduction in any medium, provided the original author(s) and source are credited.

\section{References}

1. Cleland JG, Daubert JC, Erdmann E, Freemantle N, Gras D, Kappenberger L, et al. The effect of cardiac resynchronization on morbidity and mortality in heart failure. $\mathrm{N}$ Engl $\mathrm{J}$ Med 2005;352:1539-49.

2. Abraham WT, Fisher WG, Smith AL, Delurgio DB, Leon AR, Loh $\mathrm{E}$, et al. Cardiac resynchronization in chronic heart failure. N Engl J Med 2002;346:1845-53.

3. Leclercq C, Hare JM. Ventricular resynchronization: Current state of the art. Circulation 2004;109:296-9.

4. Auricchio A, Stellbrink C, Sack S, Block M, Vogt J, Bakker P, et al. Long term clinical effect of hemodynamically optimized cardiac resynchronization therapy in patients with heart failure and ventricular conduction delay. J Am Coll Cardiol 2002;39:2026-33.

5. Butter C, Auricchio A, Stellbrink C, Fleck E, Ding J, Yu Y, et al. Effect of resynchronization therapy stimulation site on the systolic function of heart failure patients. Circulation 2001;104:3026-9.

6. Bleeker GB, Kaandorp TA, Lamb HJ, Boersma E, Steendijk P, de Roos A, et al. Effect of posterolateral scar tissue on clinical and echocardiographic improvement after cardiac resynchronization therapy. Circulation 2006;113:969-76.

7. Greenberg BH, Drew D, Botvinick EH, Werner JA, Klausner SC, Shames DM, et al. Evaluation of left ventricular performance by gated radionuclide angiography. Clin Nucl Med 1980;5:245-54.

8. Botvinick E, Frais M, O'Connell W, Faulkner D, Scheinman M, Morady F, et al. Phase image evaluation of patients with ventricular preexcitation syndromes. J Am Coll Cardiol 1984;3:799808.

9. Rosenquist M, Isaaz K, Botvinick EH, Dae M, Cockrell J, Abbott $\mathrm{J}$, et al. Relative importance of activation sequence compared to AV synchrony in left ventricular function. Am J Cardiol 1991; 67:148-56.

10. Kerwin W, Botvinick EH, O'Connell JW, Merrick SH, De Marco $\mathrm{T}$, Chatterjee $\mathrm{K}$, et al. Ventricular contraction abnormalities in dilated cardiomyopathy: Acute effects of dual chamber simultaneous biventricular pacing to correct interventricular dyssynchrony. J Am Coll Cardiol 2000;35:1221-7.

11. Botvinick E, Davis J, Dae M, O'Connell J, Schechtmann N, Abbott J, et al. Localization of ventricular tachycardia exit site and subsequent contraction sequence and functional effects with bedside radionuclide angiography. $\mathrm{J}$ Am Coll Cardiol Imaging 2008;1:605-13.

12. Harris A. Delayed development of ventricular ectopic rhythms following experimental coronary occlusion. Circulation 1950;1: $318-24$. 
13. Kelbaek H. Technetium-99m labeling of red blood cell: In vitro evaluation of a new approach. J Nucl Med 1986;27:1770-3.

14. Dekker AL, Phelps B, Dijkman B, van der Nagel T, van der Veen FH, Geskes GG, et al. Epicardial left ventricular lead placement for cardiac resynchronization therapy: Optimal pace site selection with pressure-volume loops. J Thorac Cardiovasc Surg 2004;127: 1641-7.

15. Ansalone G, Giannantoni P, Ricci R, Trambaiolo P, Fedele F, Santini M. Doppler myocardial imaging to evaluate the effectiveness of pacing sites in patients receiving biventricular pacing. J Am Coll Cardiol 2002;39:489-99.

16. Ypenburg C, Schalij MJ, Bleeker GB, Steendijk P, Boersma E, Dibbets-Schneider P. Impact of viability and scar tissue on response to cardiac resynchronization therapy in ischaemic heart failure patients. Eur Heart J 2007;28:33-41.

17. Munoz del Romeral L, Stillson C, Lesh M, Dae M, Botvinick E. The relationship of myocardial contraction and electrical excitation-the correlation between scintigraphic phase image analysis and electrophysiologic mapping. J Nucl Cardiol. doi:10.1007/ s12350-009-9114-9.

18. Fauchier L, Olivier M, Danielle CS, Dominique B, Pierre C, Fauchier JP. Interventricular and intraventricular dyssynchrony in idiopathic dilated cardiomyopathy: A prognostic study with Fourier phase analysis of radionuclide angioscintigraphy. J Am Coll Cardiol 2002;40:2022-30.
19. Pitzalis MV, Iacoviello M, Romito R, Guida P, De Tommasi E, Luzzi G, et al. Ventricular asynchrony predicts a better outcome in patients with chronic heart failure receiving cardiac resynchronization therapy. J Am Coll Cardiol 2005;45:65-9.

20. Bax JJ, Bleeker GB, Marwick TH, Molhoek SG, Boersma E, Steendijk $\mathrm{P}$, et al. Left ventricular dyssynchrony predicts response and prognosis after cardiac resynchronization therapy. J Am Coll Cardiol 2004;44:1834-40.

21. Leclercq C, Faris O, Tunin R, Johnson J, Kato R, Evans F, et al. Systolic improvement and mechanical resynchronization does not require electrical synchrony in the dilated failing heart with left bundle-branch block. Circulation 2002;106:1760-3.

22. León AR. New tools for the effective delivery of cardiac resynchronization therapy. J Cardiovasc Electrophysiol 2005;16:S42-7.

23. Young JB, Abraham WT, Smith AL, Leon AR, Lieberman R, Wilkoff $\mathrm{B}$, et al. Combined cardiac resynchronization and implantable cardioversion defibrillation in advanced chronic heart failure: The MIRACLE ICD Trial. JAMA 2003;289:2685-94.

24. Leclercq C, Kass DA. Retiming the failing heart: Principles and current clinical status of cardiac resynchronization. J Am Coll Cardiol 2002;39:194-201.

26. Conti CR. Cardiac resynchronization therapy for chronic heart failure: Why does it not always work? Clin Cardiol 2006;29:335-6. 